\title{
Recycling of Cantilevers for Nanoparticle Detection by Lift-off Technique
}

\author{
S. Merzsch ${ }^{1}$, H. S. Wasisto ${ }^{1}$, A. Waag ${ }^{1}$, I. Kirsch ${ }^{2}$, E. Uhde ${ }^{2}$, T. Salthammer ${ }^{2}$, E. Peiner ${ }^{1}$ \\ ${ }^{1}$ Braunschweig University of Technology, Institute of Semiconductor Technology, \\ Hans-Sommer-Str. 66, D-38106 Braunschweig, Germany \\ ${ }^{2}$ Fraunhofer WKI, Material Analysis and Indoor Chemistry (MAIC), \\ Bienroder Weg 54E, D-38108 Braunschweig, Germany
}

\begin{abstract}
:
Monitoring of engineered airborne nanoparticles is prerequisite to their safe industrial use. Workmen, inhaling particles, are endangered to sustain health hazards. A silicon resonant cantilever sensor has been proven a tool for sampling and recognizing airborne particles at high sensitivity. For repeated usability of the resonator the trapped particles have to be removed which is accomplished by a sacrificial layer deposited on the polluted cantilever surface. Particles, forced by an electrostatic field, settle on top of the layer in spite of the silicon. Within a wet cleaning, not only the additional layer is removed, but also the trapped nanoparticles. This method has been proven by using titanium or conventional photoresist as a covering layer. For the latter, cleaning efficiency is proven by detection of resonant frequency.
\end{abstract}

Key words: nanoparticles, cantilever, cleaning, resonant frequency

\section{Introduction}

The use of technically produced nanoparticles (NPs) is spread broadly over manifold utilizations within a variety of emerging branches of industry. Not only are the benefits considered, but also potential negative side effects. The most important issue is the impact on human health. Divers shapes and materials of NPs make it difficult to exclude possible adverse effects on organisms. Especially the detection of airborne NPs is of great importance because they are respirable by inhalation. For personal monitoring of NPs exposure resonant mass sensing methods have been proposed based on film bulk acoustic resonators (FBAR) [1], micromechanical resonators using a sensing platform [2] or a silicon cantilever driven at its fundamental or higher modes as in the present work [3].

\section{Experimental}

A cantilever-type resonator with full Wheatstone bridge piezoresistors for signal read out is applied inside a NP sampler, which was introduced more in detail elsewhere [3]. NP laden air flows through the sampler with a rate of $0.68 \mathrm{I} / \mathrm{min}$ stimulated by a fan at the back side of the sampler. An electric field $(380 \mathrm{~V})$ applied between the housing (anode) and cantilever as cathode is used to force positively charged particles towards the cantilever. Particles settle on the surface and lead to an increase of the total mass which comprises the mass of the cantilever $m_{\text {cant }}$ and the added mass $m_{\text {add }}$ by the particles. As a result of this mass increase the resonant frequencies $f_{n}$ drop according to [4]:

$$
\begin{aligned}
& \Delta f_{\mathrm{n}} \approx \frac{m_{\text {add }}}{2 m_{\text {cant }}} \\
& \Delta f_{\mathrm{n}, \min } \approx \frac{f_{\mathrm{n}}}{Q}, \Delta m_{\min } \approx \frac{2 m_{\text {cant }}}{Q}
\end{aligned}
$$

Over time, the cantilever gets crowded by particles. To enhance operating life of a sensor, brushing up by removing attached NPs has been tried in different ways. Recycling by typical wet chemical cleaning methods does not end up in cleaned unladen surfaces. An approach using silicone which can incorporate and detach the particles from flat surfaces [5] was found to fail because of the fragility of the cantilevers. The approach focused in this contribution circumvents the direct attachment of NPs to the silicon using a sacrificial layer on top of the silicon. Two different materials have been tested for usability. The first test was done with the cantilever shown in Fig. 1a. Titanium was deposited by electron beam evaporation with rotating sample. The film thickness was 
chosen to be $10 \mathrm{~nm}$ (see Fig. 1b). Particles collected by sampling stick to the titanium instead of the silicon (see Fig. 1c). Titanium can be etched selectively over silicon by buffered hydrofluoric acid (HF). After immersion for 2 min in buffered HF (6\%wt.) the layer of titanium and most of the particles have been removed from the silicon surface (see Fig. 1d).

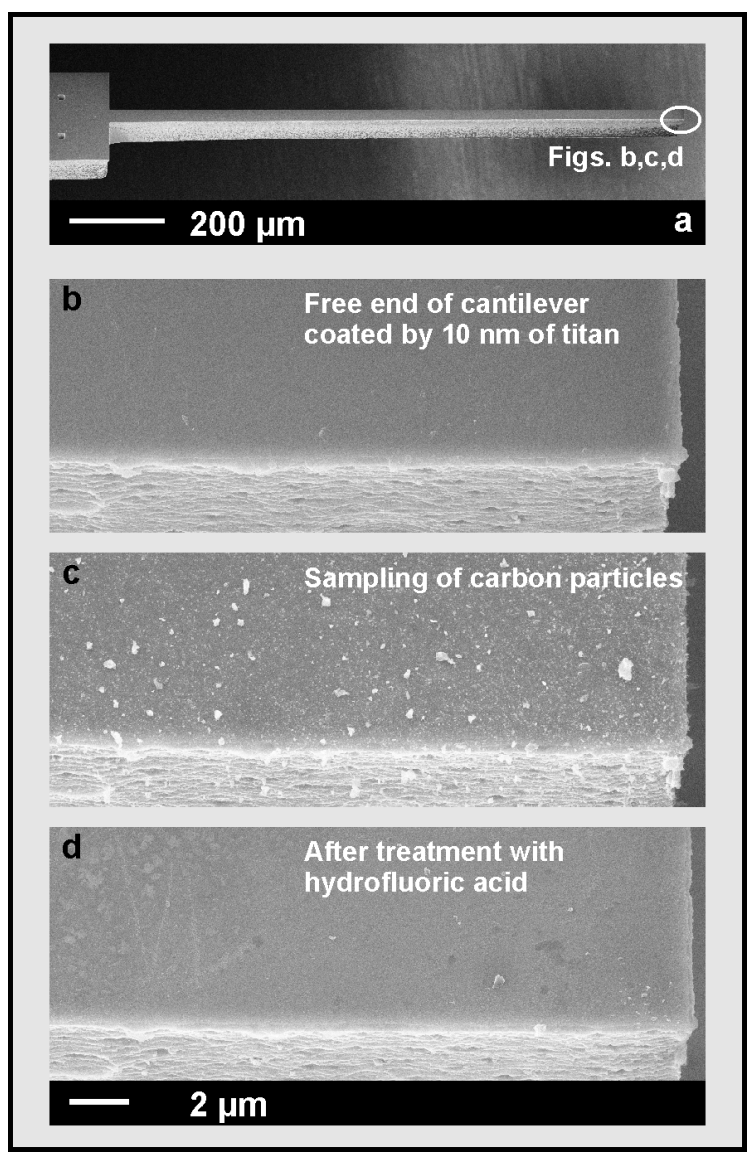

Fig. 1. Cantilever (a) for particle removal by an additional sacrificial titanium layer (b) after collection of carbon NPs (c) and after immersion in hydrofluoric acid (d) showing a high cleaning efficiency.

By counting the amount of particles, the efficiency of cleaning is about $85 \%$. Unfortunately, metal evaporation is comparatively elaborate and HF hazardous.

In the second case, the additional coating was made of photoresist. A bare cantilever (see Fig. 2a) was immersed into a solution of AZ5214 E and its thinner PGMEA (see Fig. 2b). For keeping the detection limit as high as possible, it is favorable to keep the resist layer thin and lightweight. Therefore, the ratio of volumes was chosen to be 1 part of resist to 9 parts of thinner. After immersion, the sample was cured on a hotplate for $10 \mathrm{~min}$ at $110^{\circ} \mathrm{C}$ to harden the resist (see Fig. 2c). After those pre steps, the resonator was used as a part of the mass sensing tool as usual. Carbon NP sampling inside a sealed chamber was arranged. The particles were forced by the electric field to settle at the surface as usual. Particles were trapped over the entire beam (see Fig. 2d). Because most of the area was covered by resist, there were only few particles which stuck directly to silicon. The sample has been put out again for the cleaning procedure after sampling. To recover the pure cantilever a wet chemical cleaning followed (see Fig. 2e). To slow down the reactivity of acetone at resist, it was mixed with deionized water (3:1 vol.). As long as the dissolution was ongoing, the NPs formerly sticking to the resist were able to leave the surface. The timeframe to separate was enlarged by the lower reaction speed. The lift-off was supported by motion caused by ultrasonic agitation. After $1 \mathrm{~min}$, the lift-off was stopped.
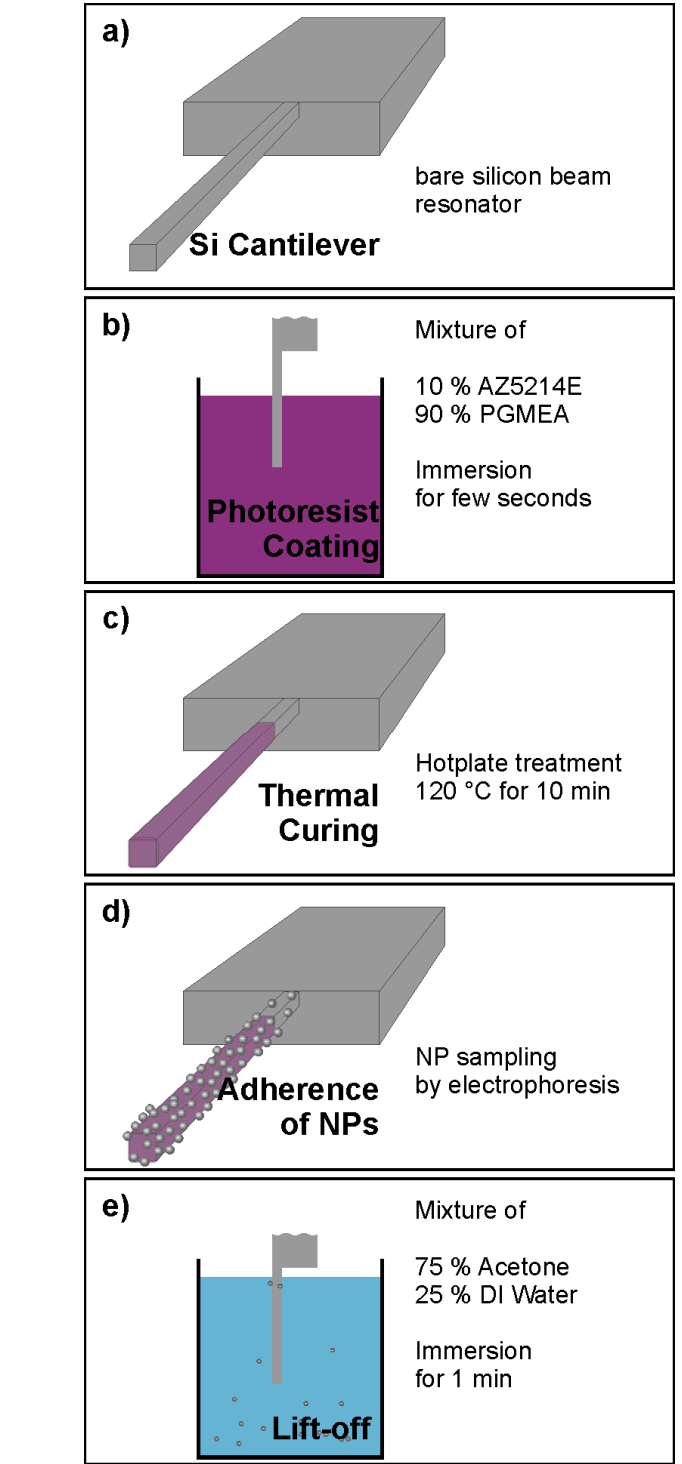

Fig. 2 Procedure for cleaning slender silicon beams (a) started using photoresist coating and curing (b, c), collecting NPs onto the sacrificial film (d) and final NPs lift-off in acetone-water enhanced by ultrasonic agitation (e). 


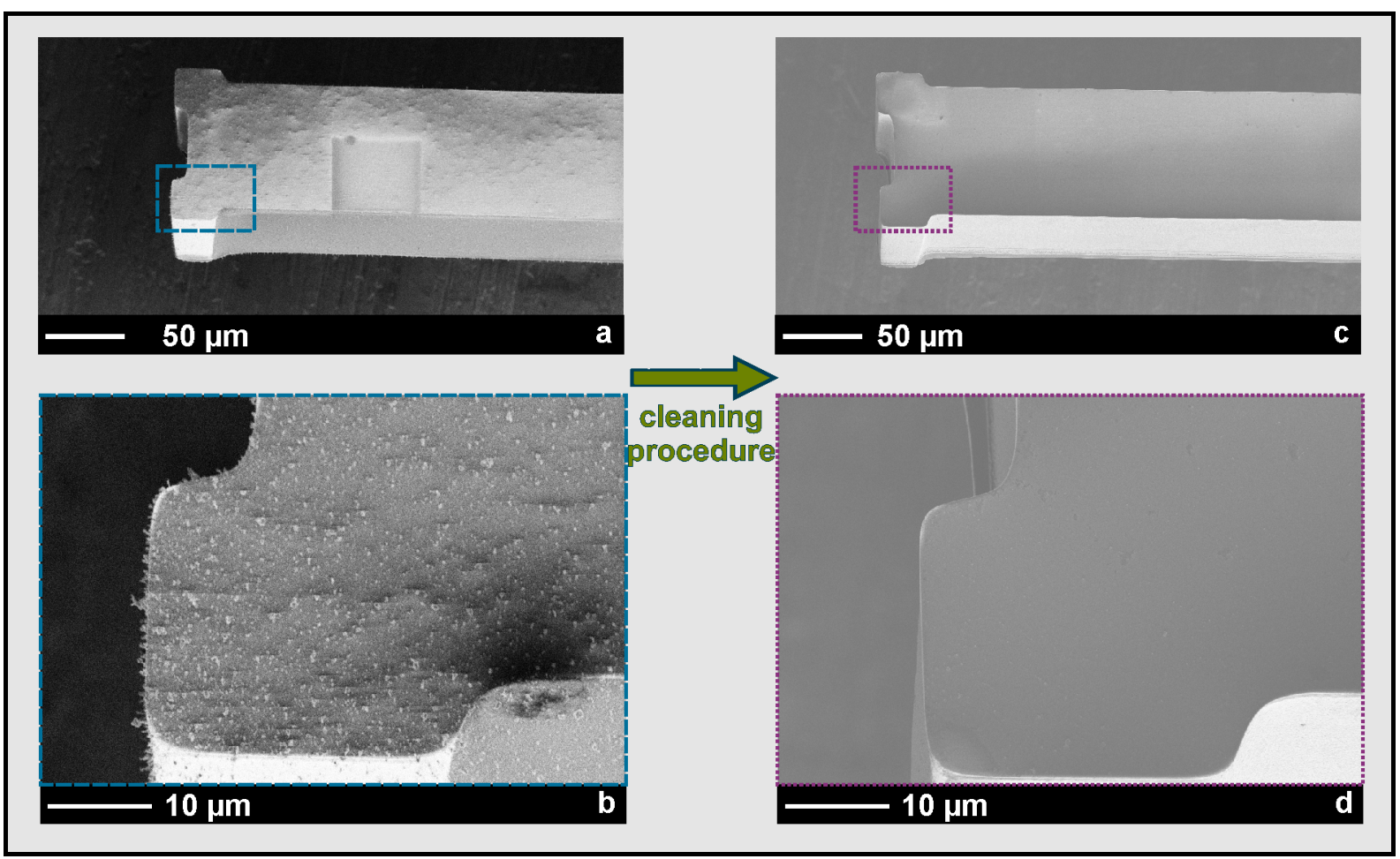

Fig. 3. Cantilever covered by a resist film and carbon NPs $(a, b)$ and after lift-off $(c, d)$.

The surface of the cantilevers free end is shown in Fig. 3 depicting carbon NPs on a homogeneous layer of resist (Figs. $3 a$ and b). Charge effects in the scanning electron microscope (SEM) photographs due to the insulating resist layer are visible. The cleaning procedure lifted most of the particles from the sensor element (Figs. 3c and d) at an efficiency reaching $98 \%$.

\section{Experiment and results}

To study the influence of the photoresist layer more precisely, the first three out-of-plane resonant frequency modes were measured. They were sampled in air with a silicon cantilever $(I=2.75 \mathrm{~mm}, w=0.1 \mathrm{~mm}, t=50 \mu \mathrm{m}$, $\left.m_{\text {cant }}=32.04 \mu \mathrm{g}\right)$ using piezoelectric actuation and piezoresistive detection (see Fig. 4).

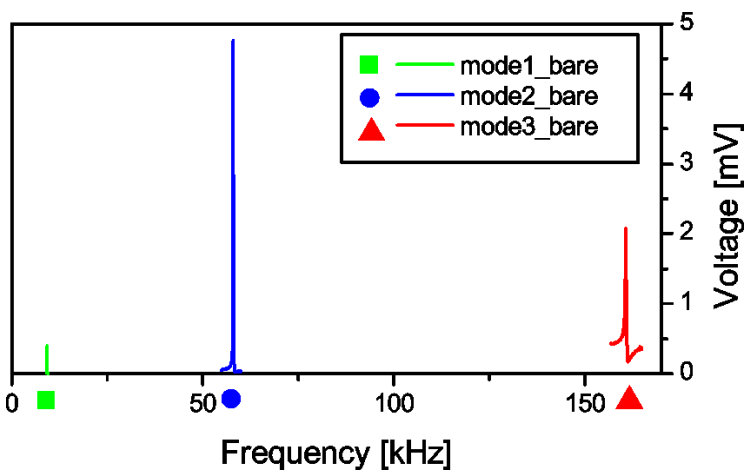

Fig. 4 Output signal of the piezoresistive strain gauge in the ranges around the lowest three resonant out-of-plane flexural modes.
Engineered carbon-based airborne NPs of a concentration of $\sim 38600 \mathrm{NP} / \mathrm{cm}^{3} \quad\left(T=23^{\circ} \mathrm{C}\right.$, $r H=30 \%, p=101.5 \mathrm{kPa}$ ) were collected on the resist-coated cantilever for $15 \mathrm{~min}$. During collection the NPs size distribution is recorded at $2-\mathrm{Hz}$ time resolution using a fast mobility particle sizer (FMPS, TSI 3091). Assuming a spherical shape of the NPs the total amount of $3.94 \times 10^{8} \mathrm{NPs}$ directed through the sampler within $15 \mathrm{~min}$ corresponds to a total NPs mass of $689.2 \mathrm{ng}$.

The resonant modes were taken again after coating, subsequent carbon particle collection and lift-off. As expected, the resonant frequencies vary according to eq. 1 (see Fig. 5 for $2^{\text {nd }}$ mode). The measured values of the resonant frequencies and quality factors are given in Fig. 6.

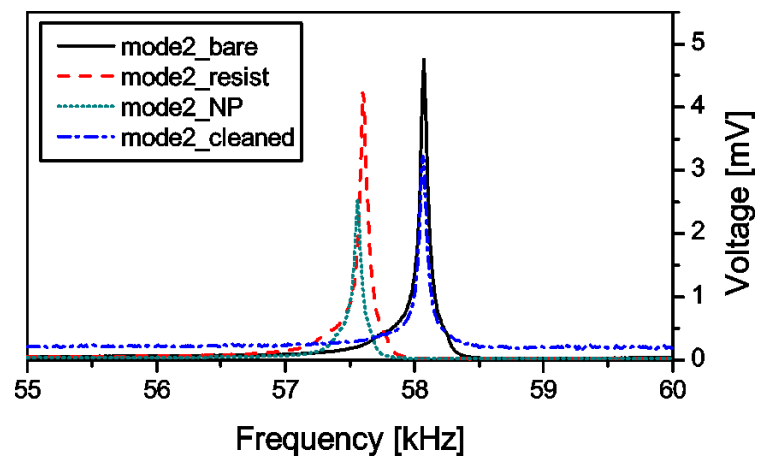

Fig. 5 Second mode measurement for different conditions of the resonator. 


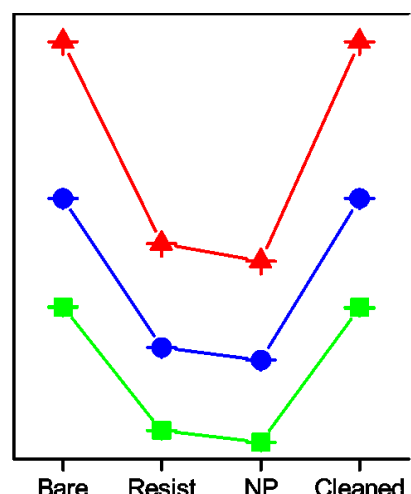

a)

Condition

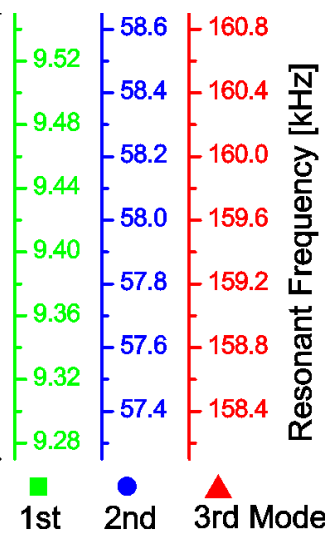

1st 2nd 3rd Mode

Fig. 6. Resonant frequency responses

Resonant frequencies and quality factors of the $2^{\text {nd }}$ mode are listed in Tab. 1.

Tab. 1: Resonant frequencies and referring quality factors for $2^{\text {nd }}$ mode.

\begin{tabular}{|c|c|c|}
\hline Condition & frequency $[\mathrm{Hz}]$ & quality factor \\
\hline Bare & $58068.78 \pm 0.17$ & $1813.86 \pm 6.1$ \\
\hline Resist & $57600.32 \pm 0.12$ & $1377.34 \pm 13.8$ \\
\hline Sampling & $57560.35 \pm 0.12$ & $1241.72 \pm 24.0$ \\
\hline Cleaned & $58068.65 \pm 0.10$ & $2292.16 \pm 88.4$ \\
\hline
\end{tabular}

Rearrangement of eq. 1 for resist mass as $m_{\text {add }}$ and neglect of effects from photoresist layer on cantilever stiffness, gives the total mass of resist covering the sensor as $516.91 \pm 0.18 \mathrm{ng}$. After sampling of carbon-based NPs, $m_{\text {add }}$ has been found to be $561.02 \pm 0.18 \mathrm{ng}$, consisting of $m_{\mathrm{NP}}$ and $m_{\text {resist }}$ as in eq. (2).

$m_{\text {add }}=m_{\text {resist }}+m_{\mathrm{NP}}$

The mass sensitivity $S$ given by eq. (3) is calculated as $0.91 \mathrm{~Hz} / \mathrm{ng}$.

$S=\frac{\Delta f}{m_{\text {add }}}$

Total mass of trapped NPs ( $m_{\mathrm{NP}}$ ) is $\sim 44.11 \mathrm{ng}$. Comparing to the total supply of $689.2 \mathrm{ng}$, $6.4 \%$ have been collected. After cleaning the frequency shift pertaining to bare cantilever is within the range of the standard deviation. Relying to the absolute values, only $0.025 \%$ of $m_{\text {add }}$ is still present after lift-off. Assuming all the resist gone, a cleaning efficiency of $99.68 \%$ for NPs mass has been achieved.

The quality factor reduces for all detected modes by coating or sampling and rises again after cleaning. The first cycle has changed the surface characteristics. This effect is assumed to be negligible for further cycles of usage.

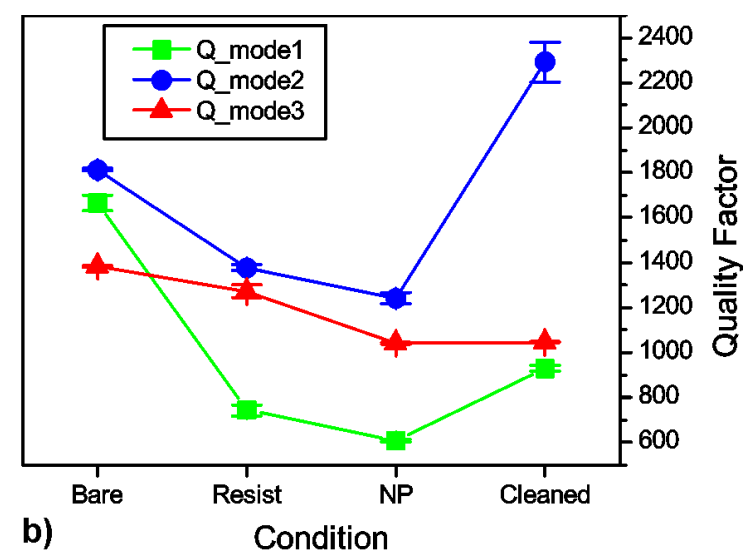

Condition

\section{Conclusions}

Prevention of direct contact between resonator body and particles by a sacrificial layer has been proven to be a valuable approach in terms of sensors life time extension. This method has reached cleaning efficiencies high above the level of conventional wet cleaning.

\section{Acknowledgements}

The authors would like to thank J. Arens and D. Rümmler for their valuable technical assistance.

This work was performed in the collaborative project "NanoExpo" funded by the German Federal Ministry of Education and Research (BMBF) within the cluster "NanoCare" under no. 03X0098A.

\section{References}

[1] I. Paprotny, F. Doering, R. White, MEMS Particulate Matter (PM) Monitor for Cellular Deployment, IEEE Sensors 2010, 2435-2440 (2010); doi: 10.1109/ICSENS.2010.5690758

[2] A. Hajjam, J.C. Wilson, A. Rahafrooz and S. Pourkamali, Fabrication and characterization of thermally actuated micromechanical resonators for airborne particle mass sensing: II. Device fabrication and characterization, J. Micromech. Microeng. 20, 125019 (10pp) (2010); doi: 10.1088/0960-1317/20/12/125019

[3] S. Merzsch, H.S. Wasisto, A. Waag, I. Kirsch, E. Uhde, T. Salthammer, E. Peiner, Low-weight Electrostatic Sampler for Airborne Nanoparticles, Proc. IEEE Sensors Conf. 2011, 1177-1180 (2011); doi: 10.1109/ICSENS.2011.6127335

[4] K. Naeli and O. Brand, Cancellation of environmental effects in resonant mass sensors based on resonance mode and effective mass, Rev. Sci. Instrum. 80, 063903 (2009); doi: $10.1063 / 1.3143567$

[5] S. Merzsch, H.S. Wasisto, A. Waag, I. Kirsch, E. Uhde, T. Salthammer, E. Peiner, Cleaning of structured templates from nanoparticle accumulation using silicone, Microsyst. Technol., (2012); doi: 10.1007/s00542-011-1379-7 